\title{
ОБОБЩЕННО-ПЕРИОДИЧЕСКИЕ ДВИЖЕНИЯ ДИНАМИЧЕСКИХ И НЕАВТОНОМНЫХ ПЕРИОДИЧЕСКИХ СИСТЕМ
}

\author{
А.П. Афранасъев, д.фр.-м.н., профрессор, ара@isa.ru \\ (Институт проблем передачи информаиии им. А.А. Харкевича РАН (ИППИ РАН), \\ Большой Каретный пер., 19, стр. 1, г. Москва, 127051, Россия; \\ Московский государственный университет им. М.В. Аомоносова, \\ Аенинские горы, 1, г. Москва, 119991, Россия; \\ Наииональный исследовательский технологический университет "МИСиС", \\ Аенинский просп., 6, г. Москва, 119049, Россия; \\ НИУ "Высшая школа экономики", ул. Мясниикая, 20, г. Москва, 101000, Россия); \\ C.M. Дзюба, ә.фр.-м.н., профрессор, sdzyuba@mail.ru; \\ И.И. Емельянова, ст. преподаватель, етеlyanova-123@yandex.ru \\ (Тверской государственный технический университет, \\ наб. Аф. Никитина, 22, г. Тверь, 170026, Россия)
}

Настоящий обзор посвящен истории изучения ситуации общего положения движений динамических и неавтономных периодических систем, поскольку, как оказалось, полное и подробное описание ситуации общего положения позволяет решить проблему построения обобщенно-периодических движений динамических и неавтономных периодических систем. Необходимость численного изучения указанных систем объясняется тем, что подавляющее большинство моделей реальных технических, биологических, экономических и др. процессов описываются именно такими системами.

В автономном случае обобщенно-периодическое движение эквивалентно классическому рекуррентному движению, введенному и изученному еще Дж. Биркгофом. С понятием рекуррентного движения прямо связано понятие минимального множества. Именно эти два понятия и определяют ситуацию общего положения классических динамических систем.

Особое значение построение и исследование рекуррентных движений и минимальных множеств приобрели в связи с потребностями хаотической динамики и гиперболической теории. Однако еще до недавнего времени отсутствовали общие методы построения рекуррентных движений и минимальных множеств: все сводилось к построению аттракторов отдельных систем дифференциальных уравнений с полилинейной правой частью. Открытие же понятия обобщенно-периодического движения привело к созданию общего метода построения и исследования всех минимальных множеств, содержащихся в предельных множествах динамических систем. Более того, понятие обобщенно-периодического движения позволило перенести все основные понятия классической теории динамических систем на неавтономные периодические системы и описать ситуацию общего положения в таких системах с единых позиций. Соответственно, появилась возможность прямого переноса метода построения рекуррентных движений на построение обобщенно-периодических движений неавтономных периодических систем, что позволило выполнять численное построение таких движений с единых позиций.

Ключевые слова: динамические и неавтономные периодические системы, ситуачия общего положения, обобщенно-периодические движения, построение обобщенно-периодических движений.

До недавнего времени отсутствовали какиелибо общие методы и алгоритмы построения рекуррентных решений и минимальных множеств автономных систем обыкновенных дифференциальных уравнений. Как оказалось, причина в том, что ситуация общего положения решений не была достаточно изучена.

Что же мы понимаем под ситуацией общего положения? Для ответа на этот вопрос рассмотрим нормальную систему дифференциальных уравнений, векторная запись которой имеет вид

$$
x^{\prime}=f(t, x),
$$

где $x=\left(x^{1}, \ldots, x^{n}\right)$ - векторная функция действительного переменного $t$, а $f=\left(f^{1}, \ldots, f^{n}\right)$ - векторная функция, определенная и непрерывная вместе со своими частными производными $\frac{\partial f^{i}}{\partial x^{j}}, i, j=1, \ldots, n$, на прямом произведении $R \times R^{n}$ действительной оси $R$ и евклидова векторного пространства $R^{n}$.
Кроме того, предположим, что $f$-Т-периодическая по $t$ функция.

Если $n=2$, то справедлива следующая теорема Х.Л. Массера [1]: пусть каждое решение $\xi(t)$ системы (1) определено для всех $t \geq t_{0}\left(t \leq t_{0}\right)$; тогда, если (1) имеет решение, ограниченное при этих значениях $t$, данная система имеет Т-периодическое решение $\varphi(t)$. Согласно [1], это утверждение справедливо и для линейных систем произвольного порядка. Однако в нелинейном случае теорема Массера неверна уже при $n=3$ [2].

Задолго до появления теоремы Массера для гладких автономных систем

$$
\dot{x}=f(x)
$$

Дж. Биркгофом было введено определение рекуррентного решения. Данное решение содержит в себе как частный случай периодическое решение. При этом из существования ограниченного решения всегда следует существование рекуррентного 
решения и наоборот. Именно эту ситуацию мы и называем ситуацией общего положения решений автономных систем.

Свойства решений системы (1), вообще говоря, несколько отличаются от свойств решений системы (2). Так, например, в неавтономном случае траектории решений могут пересекаться. Это приводит к тому, что определение рекуррентности на неавтономный случай прямо не переносится. Отсюда следует, что для неавтономной системы вида (1) при $n>2$ ситуация общего положения периодическим решением не определена, а рекуррентным решением не определяется (ввиду отсутствия определения последнего).

Исследования, выполненные в работах [3-5], привели к определению обобщенно-периодического решения [2]. Оказалось, что из существования ограниченного решения всегда следует существование обобщенно-периодического решения. Более того, в автономном случае это определение эквивалентно определению рекуррентного решения. Другими словами, обобщенно-периодическое решение дает ситуацию общего положения и в автономном, и в неавтономном случаях.

Выясним историческую роль и место ситуации общего положения в теории динамических систем.

Хорошо известно, что геометрическое исследование А. Пуанкаре «О кривых, определяемых дифференциальными уравнениями» [6], а также его знаменитый мемуар «Новые методы небесной механики» $[7,8]$ в сочетании с диссертацией А.М. Ляпунова [9] заложили в конце девятнадцатого века основы качественной теории дифференциальных уравнений.

На взгляд авторов, особого упоминания заслуживает мемуар А. Пуанкаре «Об одной геометрической теореме» - прощальный поклон и завещание великого ученого [8]. Сформулированная здесь в виде гипотезы теорема о неподвижной точке некоторого гладкого отображения кругового кольца в себя была доказана Дж. Биркгофом уже после смерти А. Пуанкаре. Трудно утверждать наверняка, но, возможно, именно работа над этой теоремой окончательно укрепила силы и дух Дж. Биркгофа и привела его к дальнейшим исследованиям по теории динамических систем и топологии. Во всяком случае современники утверждали, что «настоящим учителем Биркгофа был Пуанкаре» и что «Биркгоф усердно изучал все работы Пуанкаре по динамике» (например [8]).

Каково же отношение Дж. Биркгофа к общей теории динамических систем? Ответ на этот вопрос прост - он ее создал.

В основе общей теории динамических систем лежит понятие движения. В случае автономной системы (2) движение эквивалентно решению, определенному на всей оси $R$. В случае абстрактной динамической системы, характеризуемой однопараметрической группой преобразований, движение естественным образом обобщает понятие решения.

Конечной целью общей теории динамических систем является определение всех возможных типов движений и установление связи между ними. В [10] введены и подробно изучены такие фундаментальные понятия, как $\alpha$ - и $\omega$-предельные точки и множества, возвращаемость областей и центральные движения, минимальные множества и рекуррентные движения. Свои результаты Биркгоф дополнил знаменитой эргодической теоремой, заложив, таким образом, основы эргодической теории.

Нельзя сказать, что книга Дж. Биркгофа написана простым и понятным языком, особенно для своего времени: об этом в своих воспоминаниях говорил даже Л.С. Понтрягин. Однако уже в середине прошлого века она нашла последователей и получила развитие в работах А.А. Маркова, Г.Ф. Хильми, В.В. Немыцкого, В.В. Степанова, А.Н. Колмогорова и многих других математиков (например в [11]).

Наряду с работами по общей теории динамических систем качественная теория дифференциальных уравнений одновременно получила более чем продвинутое развитие в области систем с интегральным инвариантом. Полученные в этом направлении результаты К. Каратеодори и А.Я. Хинчина существенным образом дополнили и обобщили теорему Пуанкаре о возвращении.

Наряду с упомянутыми работами в качественной теории дифференциальных уравнений появилось и совершенно новое важное направление структурная устойчивость, изначально названная грубостью динамических систем. Основоположниками в этой области выступили А.А. Андронов и Л.С. Понтрягин, создавшие теорию грубых систем на плоскости [12-15]. Ими, в частности, было доказано, что грубые системы всюду плотны на множестве двумерных систем.

Перенесение понятия грубости уже на трехмерный случай оказалось существенно осложненным. Объясняется это гораздо менее тривиальным характером фазовых портретов грубых трехмерных систем. Принципиально важным оказалось то, что здесь впервые был построен пример грубого диффеоморфизма со счетным множеством седловых предельных циклов, имеющих неограниченно возрастающий период [16].

Существенным шагом вперед в этом (и не только в этом) направлении стала книга [17] (см. также [18]). В ней наиболее привлекают не установленная структурная устойчивость геодезических потоков на замкнутых римановых многообразиях отрицательной кривизны, а четкое описание вида и установление свойств динамических и дискретных систем, названных впоследствии У-системами Д.В. Аносова.

Аносов показал, что каждая У-система структурно устойчива. Более того, оказалось, что структурная устойчивость таких систем неизбежно соче- 
тается с экспоненциальным разбеганием фазовых кривых и перемешиванием.

Особый интерес к этому открытию подогрела появившаяся в начале 60-х годов прошлого века следующая попытка построения теории турбулентного движения жидкости, непосредственно приводящая к понятию гиперболического притягивающего множества (например [18]).

Следуя [18], рассмотрим замкнутый сосуд, заполненный несжимаемой вязкой жидкостью, приводимой в движение внешней силой - мешалкой. Динамику движения жидкости описывают уравнениями Навье-Стокса. Точками фазового пространства такой системы являются бездивергентные векторные поля скоростей жидкости, в которых положения равновесия представляют собой стационарные поля скоростей. Циклы же здесь отождествляют с периодическими движениями жидкости, в которых скорость движения в каждой точке пространства меняется по периодическому закону.

Если вязкость жидкости достаточно велика, то система уравнений Навье-Стокса имеет единственное положение равновесия, к которому притягиваются все фазовые кривые. Такое движение жидкости называют ламинарным, и всякое другое течение под действием вязкости со временем стремится к нему.

С уменьшением вязкости ламинарное движение может потерять устойчивость, что может привести к образованию структурно устойчивого предельного цикла. Дальнейшее уменьшение вязкости, вообще говоря, может вызвать потерю устойчивости данного цикла. При этом возможно появление из цикла некоторого другого, более сложного непериодического движения, притягивающего соседние.

Гипотеза математического описания турбулентности состоит в том, что в бесконечномерном фазовом пространстве имеется конечномерное многообразие или множество, к которому притягиваются все фазовые кривые. Само же притягивающее множество должно представлять собой фазовое пространство или У-системы, или системы, качественно близкой к ней: в любом случае здесь должны сохраняться экспоненциальное разбегание траекторий и следующее из него перемешивание.

С физической точки зрения данная гипотеза выглядит весьма правдоподобно и подтверждается многочисленными экспериментальными исследованиями. Однако, несмотря на все прилагаемые усилия превратить ее в теорему, насколько авторам известно, в настоящее время так и не удалось.

Оставив в стороне вопросы структурной устойчивости, заметим, что с этого времени взгляд на теорию динамических систем постепенно начал претерпевать изменения. В наступившей эпохе гипермодернизма понятия рекуррентного движения и минимального множества постепенно стали исчезать из употребления, а о ситуации общего положе- ния вообще перестали говорить. Так, например, попытка отыскать аналог рекуррентного движения для неавтономных систем фактически сводилась к изучению траекторий дискретных динамических систем [19].

В работах $[20,21]$ для изучения ситуации общего положения в абстрактных автономных динамических и неавтономных периодических системах был предложен метод, существенным образом использующий только групповые свойства оператора сдвига вдоль движений этих систем. В основе данного метода, собственно, и лежит определение движения, которое будем называть обобщенно-периодическим.

Существование в любой из указанных систем движения при $t \geq t_{0}$, расположенного в некотором компактном множестве, влечет за собой существование обобщенно-периодического движения. Оказалось, что в автономном случае определение равномерно обобщенно-периодического движения эквивалентно определению рекуррентного движения. При этом определение равномерно устойчивого движения наиболее точно передает характер последнего и, кроме того, устанавливает обязательный (причем достаточно жесткий) сценарий асимптотического перехода произвольного движения к рекуррентному. Последнее позволило в работах $[22,23]$ осуществить разработку общего метода построения рекуррентных движений и минимальных множеств классических динамических систем. Более того, как показано в работе [24], данный метод без каких-либо существенных изменений переносится на проблему построения обобщенно-периодических движений неавтономных периодических систем.

Заметим, что изучение ситуации общего положения и введение понятия обобщенно-периодического движения дают новые характеристические свойства рекуррентных движений, дополняющие классические [22, 24]. Эти новые свойства дают ряд дополнительных возможностей для исследования динамических систем. Проиллюстрируем их на следующих примерах.

Пример 1. Важнейшими из всех качественных типов движений, как известно, являются устойчивые по Пуассону и рекуррентные движения. Отличительной особенностью этих движений является то, что каждое рекуррентное движение устойчиво по Пуассону, но не наоборот. При этом из существования движения, положительно или отрицательно устойчивого по Лагранжу, следует существование, по крайней мере, одного рекуррентного движения. Однако из существования рекуррентного движения не следует существование устойчивого по Пуассону, но нерекуррентного движения. В настоящее время связь между рекуррентными и устойчивыми по Пуассону нерекуррентными движениями изучена на уровне определений [25], а разработанная в [3-5, 20-24] техника позволяет 
осуществить более полное установление такой связи.

Напомним, что множество $M$ называется минимальным, если оно непусто, замкнуто, инвариантно и не содержит ни одного собственного подмножества, обладающего этими тремя свойствами. Заметим также, что в компактном метрическом пространстве движений замыкание траектории рекуррентного движения является компактным минимальным множеством $M$ и наоборот. При этом замыкание $H$ траектории устойчивого по Пуассону движения называется квазиминимальным множеством.

Каждое квазиминимальное множество $H$ компактно и содержит компактное минимальное множество $M$. Однако $M$ не обязано быть собственным подмножеством множества $H$. Именно случай, в котором $M$ является собственным подмножеством множества $H$, и определяет ситуацию существования устойчивого по Пуассону нерекуррентного движения.

Как показано в работе [22], в квазиминимальном множестве $H$, не являющемся минимальным множеством, объединение всех минимальных множеств компактно и нигде не плотно в $H$. При этом точки, лежащие на траекториях нерекуррентных устойчивых по Пуассону движений, образуют множество второй категории типа $G_{\delta}$, то есть его дополнение может быть представлено как объединение счетного числа замкнутых множеств, нигде не плотных в $H$. Более полно, согласно теореме Хильми, в квазиминимальном множестве $H$, не являющемся минимальным множеством, точки, лежащие на траекториях нерекуррентных устойчивых по Пуассону движений и всюду плотных в $H$, образуют множество второй категории типа $G_{\delta}$. Несложно также показать, что каждое такое квазиминимальное множество $H$ содержит несчетное множество нерекуррентных устойчивых по Пуассону движений, всюду плотных в $H$. Как оказалось, все это имеет большое значение при численном исследовании уравнений хаотической динамики, в частности, при построении странных аттракторов (см. пример 3).

Пример 2. Системы с инвариантной мерой, как известно, обладают рядом свойств, выгодно выделяющих их из всего множества динамических систем. Важнейшее из этих свойств состоит в том, что, согласно теореме о возвращении точек Пуанкаре-Каратеодори, для систем, имеющих в сепарабельном пространстве положительную конечную инвариантную меру, почти все точки пространства устойчивы по Пуассону. Как показано в монографии [2], применение метода, использовавшегося при описании ситуации общего положения, позволяет существенно уточнить этот классический результат. Именно в условиях теоремы Пуанкаре-Каратеодори каждая устойчивая по Пуассону точка является точкой какого-либо минимального мно- жества, то есть почти все точки пространства являются точками минимальных множеств.

Пример 3. В последнее время при исследовании уравнений хаотической динамики стали широко применяться численные методы. В частности, почти все известные на сегодняшний день странные аттракторы построены именно численно.

Для определенности рассмотрим аттрактор Лоренца $[14,23]$. Согласно многочисленным вычислительным экспериментам многих исследователей, данный аттрактор состоит из трех положений равновесия (один седлоузел и два седлофокуса) и множества незамкнутых устойчивых по Пуассону траекторий, всюду плотно заполняющих этот аттрактор (за исключением седлофокусов). Более того, сам аттрактор представляет собой объединение трех положений равновесия и замыкания устойчивой по Пуассону траектории. При этом считается также, что аттрактор содержит счетное всюду плотное множество седловых предельных циклов [14]. Проведенные авторами численные эксперименты показали, что данная гипотеза нуждается в существенной доработке.

Действительно, как отмечалось в примере 1, в квазиминимальном множестве $H$, не являющемся минимальным множеством, объединение всех минимальных множеств компактно и нигде не плотно. Поэтому, если аттрактор Лоренца имеет указанную выше структуру относительно положений равновесия и устойчивых по Пуассону траекторий, он не может содержать счетного всюду плотного множества седловых предельных циклов. Более того, как показано в [23], с точностью до ошибки вычислений можно утверждать, что в системе Лоренца имеются лишь три указанных выше положения равновесия и минимальное множество, образованное незамкнутым рекуррентным движением. Все эти данные, полученные специально разработанным численным методом, хорошо согласуются с теоретическими результатами, приведенными в $[22,24]$.

\section{Литература}

1. Massera J.L. The Existence of Periodic Solutions of Systems of Differential Equations. Duke Math. J., 1950, vol. 17, pp. $457-475$

2. Афанасьев А.П., Дзюба С.М. Устойчивость по Пуассону в динамических и непрерывных периодических системах. М.: ЛКИ, 2007. $240 \mathrm{c.}$

3. Афанасьев А.П., Дзюба С.М. К вопросам управления в периодических процессах // Изв. РАН. Теория и системы управления. 1998. № 4. С. 15-20.

4. Дзюба С.М. Два многомерных дополнения к теореме Пуанкаре-Бендиксона // Дифференц. уравнения. 1995. Т. 31. № 4. С. 579-582.

5. Дзюба С.М. Об условно-периодических решениях дифференциальных уравнений // Дифференц. уравнения. 1999. T. 35. № 8. С. 1020-1023.

6. Пуанкаре А. О кривых, определяемых дифференциальными уравнениями. М.-Л.: ОГИЗ, 1947. 256 с.

7. Пуанкаре А. Избранные научные труды. М.: Наука, 1971. T. $1.772 \mathrm{c}$ 
8. Пуанкаре А. Избранные научные труды. М.: Наука, 1972. T. 2. $1000 \mathrm{c}$

9. Ляпунов А.М. Общая задача об устойчивости движения. М.: Изд-во ОНТИ, 1935. 174 с.

10. Биркгоф Дж. Динамические системы. М.-Л.: ОГИЗ, $1941.332 \mathrm{c}$.

11. Немыцкий В.В., Степанов В.В. Качественная теория дифференциальных уравнений. М.: Едиториал УРСС, 2004. 550 с.

12. Андронов А.А., Витт А.А., Хайкин С.Э. Теория колебаний. М.: Физматгиз, 1959. 550 с.

13. Андронов А.А., Понтрягин Л.С. Грубые системы // Докл. АН СССР. 1937. Т. 14. № 5. С. 247-251.

14. Баутин И.Н., Леонтович Е.А. Методы и приемы качественного исследования динамических систем на плоскости. М.: Наука, 1990. 486 с.

15. Понтрягин Л.С. Избранные научные труды. М.: Наука, 1988. T. 2. $576 \mathrm{c}$

16. Смейл С. Структурно устойчивый дифференцируемый гомеоморфизм с бесконечным числом периодических точек. Киев: Изд-во Ин-та матем. АН УССР, 1961. 426 с.

17. Аносов Д.В. Геодезические потоки на замкнутых римановых многообразиях отрицательной кривизны // Тр. МИАН. 1967. № 9. C. 3-24.
18. Арнольд В.И. Дополнительные главы теории обыкновенных дифференциальных уравнений. М.: Наука, 1978. $304 \mathrm{c}$.

19. Хейл Дж. Теория функционально-дифференциальных уравнений. М.: Мир, 1984. 421 с.

20. Афанасьев А.П., Дзюба С.М. Периодический оператор сдвига и квазипериодические кривые // Дифференц. уравнения. 2004. Т. 40. № 10. С. 1367-1372.

21. Афанасьев А.П., Дзюба С.М. О рекуррентных траекториях, минимальных множествах и квазипериодических движениях динамических систем // Дифференц. уравнения. 2005. T. 41. № 11. C. 1469-1474.

22. Афанасьев А.П., Дзюба С.М. Метод построения минимальных множеств динамических систем // Дифференц. уравнения. 2015. Т. 51. № 7. С. 835-841.

23. Афанасьев А.П., Дзюба С.М. Построение минимальных множеств динамических систем полиномиальной правой частью // Дифференц. уравнения. 2015. Т. 51. № 11. С. 1411-1419.

24. Афанасьев А.П., Дзюба С.М. Обобщенно-периодические движения неавтономных систем // Дифференц. уравнения. 2017. T. 53. № 1. C. 3-9.

25. Cheban D.N. Asymptotically almost periodic solutions of differential equations, HPC, NY, 2009, 204 p.

\title{
GENERALIZED PERIODIC MOTIONS OF DYNAMICAL AND NON-AUTONOMOUS PERIODIC SYSTEMS
}

\author{
A.P. Afanasiev ${ }^{1,2,3,4}$, Dr.Sc. (Physics and Mathematics), Professor, apa@isa.ru \\ S.M.Dzyuba ${ }^{5}$,Dr.Sc. (Physics and Mathematics), Professor, sdzyuba@mail.ru \\ I.I. Emelyanova ${ }^{5}$, Senior Lecturer, emelyanova-123@yandex.ru \\ ${ }^{1}$ Institute for Information Transmission Problems of the Russian Academy of Sciences, IITP RAS, \\ Bolshoy Karetny Lane 19, bldg. 1, Moscow, 127994, Russian Federation \\ ${ }^{2}$ Lomonosov Moscow State University, Leninskie Gory, Moscow, 119991, Russian Federation \\ ${ }^{3}$ National University of Scince Research Technological "MISIS", \\ Leninsky Ave. 6, Moscow, 119049, Russian Federation \\ ${ }^{4}$ National Research University, Higher School of Economics, Myasnitskaya St. 20, Moscow, 101000, Russian Federation \\ ${ }^{5}$ Tver State Technical University, Nikitin Quay 22, Tver, 170026, Russian Federation
}

Abstract. This review represents the history of study analysis of the situation of typical behavior of dynamical and nonautonomous periodic system motions. The necessity of this investigation is due to the fact that the complete and detailed description of a typical behavior situation makes it possible to solve the problem of construction of generalized periodic motions of dynamical and non-autonomous periodic systems. The necessity of numerical investigation of indicated systems is due to the fact that the vast majority of real technological, biological, economical and others process models are described by these specific systems.

In the autonomous case a generalized periodic motion is equivalent to a classical recurrent motion, which was introduced and investigated by G. Birkhoff. The concept of the recurrent motion is related to the concept of a minimal set. These two concepts define the situation of typical behavior of classical dynamical systems.

The construction and investigation of recurrent motions and minimal sets took on special significance due to the requirements of chaotic dynamics and the hyperbolic theory. However, until quite recently there were no general methods of constructing recurrent motions and minimal sets. It came down to constructing attractors of separate systems of differential equations with a polylinear right part. The discovery of the generalized periodic motion concept resulted in creation of the general method of construction and investigation of all minimal sets contained in limit sets of dynamical systems. Furthermore, the concept of a generalized periodic motion allowed adapting all the basic definitions of the classical dynamical system theory to non-autonomous periodic systems and describing the situation of typical behavior in such systems on common ground. Therefore, it allows adapting directly the method of recurrent motion construction to the construction of generalized periodic motions of non-autonomous periodic systems that made the numerical construction of such motions on common ground possible.

Keywords: dynamical and non-autonomous periodic systems, situation of typical behavior, generalized-periodic motions, construction of generalized-periodic motions. 


\section{References}

1. Massera J.L. The Existence of Periodic Solutions of Systems of Differential Equations. Duke Math. J. 1950, vol. 17, pp. 457-475.

2. Afanasiev A.P., Dzyuba S.M. Ustoychivost po Puassonu v dinamicheskikh i nepreryvnykh periodicheskikh systemakh [Poisson stability in dynamic and indiscrete periodic systems]. Moscow, LKI Publ., 2007, 240 p.

3. Afanasiev A.P., Dzyuba S.M. On the management question in periodic processes. Izv. RAN. Teoriya i sistemy upravleniya [Jour. of Computer and Systems Sciences Int.]. 1998, no. 4, pp. 15-20 (in Russ.)

4. Dzyuba S.M. Two multidimensional supplements to the Poincare-Bendixson theorem. Differents. uravneniya [Differential Equations]. 1995, vol. 31, no. 4, pp. 579-582 (in Russ.).

5. Dzyuba S.M. On conditionally periodic solutions of differential equations. Differents. uravneniya [Differential Equations]. 1999, vol. 35, no. 8, pp. 1020-1023 (in Russ.).

6. Poincare J.H. O krivykh, opredelyaemykh differentsialnymi uravneniyami [On Curves That are Defined by Differential Equations]. Moscow, L., OGIZ Publ., 1947, 256 p.

7. Poincare J.H. Izbrannye nauchnye trudy [Selected Scientific Papers]. Moscow, Nauka Publ., 1971, vol. 1, 772 p.

8. Puankare J.H. Izbrannye nauchnye trudy [Selected Scientific Papers]. Moscow, Nauka Publ., 1972, vol. 2, 1000 p.

9. Lyapunov A.M. Obshchaya zadacha ob ustoychivosti dvizheniya [A General Dynamical Stability Problem]. Moscow, ONTI Publ., 1935, 174 p.

10. Birkgof Dzh. Dinamicheskie sistemy [Dynamic System]. Moscow, L., OGIZ Publ., 1941, 332 p.

11. Nemytsky V.V., Stepanov V.V. Kachestvennaya teoriya differentsialnykh uravneny [Qualitative Theory of Differential Equations]. Moscow, Editorial URSS Publ., 2004, 550 p.

12. Andronov A.A., Vitt A.A., Khaykin S.E. Teoriya kolebany [Oscillation Theory]. Moscow, Fizmatgiz Publ., 1959, $550 \mathrm{p}$.

13. Andronov A.A., Pontryagin L.S. Coarse systems. Dokl. AN SSSR [Proc. USSR Science Academy]. 1937, vol. 14, no. 5, pp. 247-251 (in Russ.).

14. Bautin I.N., Leontovich E.A. Metody i priemy kachestvennogo issledovaniya dinamicheskikh sistem na ploskosti [Methods and Procedures of Qualitative Research on Dynamic Systems on a Plane]. Moscow, Nauka Publ., 1990, 486 p.

15. Pontryagin L.S. Izbrannye nauchnye trudy [Selected Scientific Papers]. Moscow, Nauka Publ., 1988, vol. 2,576 p.

16. Smeyl S. Strukturno ustoychivy differentsiruemy gomeomorfizm s beskonechnym chislom periodicheskikh tochek [Structurally Stable Differentiable Homeomorphism with an Infinite Number of Periodic Points]. Kiev, In-t matematiki AN USSR Publ., 1961, 426 p.

17. Anosov D.V. Geodezicheskie potoki na zamknutykh rimanovykh mnogoobraziyakh otritsatelnoy krivizny [Geodesic Flows on Closure Riemann's Manifolds of Negative Curvature]. Moscow, Tr. MIAN Publ., 1967, no. 9, pp. 3-24 (in Russ.).

18. Arnold V.I. Dopolnitelnye glavy teorii obyknovennykh differentsialnykh uravneny [Additional Chapters of the Ordinary Differential Equation Theory]. Moscow, Nauka Publ., 1978, 304 p.

19. Hale J. Theory of Functional Differential Equations. Springer Publ., 1977 (Russ. ed.: Moscow, Mir Publ., 1984, 421 p.).

20. Afanasiev A.P., Dzyuba S.M. Periodic translation operators and quasiperiodic curves. Differents. uravneniya [Differential Equations]. 2004, vol. 40, no. 10, pp. 1367-1372 (in Russ.).

21. Afanasiev A.P., Dzyuba S.M. On recurrent trajectories, minimal sets, and quasiperiodic motions of dynamical systems. Differents. uravneniya [Differential Equations]. 2005, vol. 41, no. 10, pp. 1469-1474.

22. Afanasiev A.P., Dzyuba S.M. Method for constructing minimal sets of dynamical systems. Differents. uravneniya [Differential Equations]. 2015, vol. 51, no. 7, pp. 835-841 (in Russ.).

23. Afanasiev A.P., Dzyuba S.M. Construction of the minimal sets of differential equations with polynomial right-hand side. Differents. uravneniya [Differential Equations]. 2015, vol. 51, no. 11, pp. 1411-1419 (in Russ.).

24. Afanasiev A.P., Dzyuba S.M. Generalized-periodic motions of nonautonomous systems. Differents. uravneniya [Differential Equations]. 2017, vol. 53, no. 1, pp. 3-9 (in Russ.).

25. Cheban D.N. Asymptotically Almost Periodic Solutions of Differential Equations. HPC, NY, 2009, 204 p.

\section{Примеры бибциографического описания статьи}

1. Афанасьев А.П., Дзюба С.М., Емельянова И.И. Обобщенно-периодические движения динамических и неавтономных периодических систем // Программные продукты и системы. 2017. T. 30. № 2. C. 235-240; DOI: 10.15827/0236-235X.118.235-240.

2. Afanasiev A.P., Dzyuba S.M., Emelyanova I.I. Generalized periodic motions of dynamical and non-autonomous periodic systems. Programmnye produkty i sistemy [Software \& Systems]. 2017, vol. 30, no. 2, pp. 235-240 (in Russ.); DOI: 10.15827/0236-235X.118.235-240. 